Ann. Biol. anim. Bioch. Biophys., I974, 14 (4-B), 747-755.

\title{
RÔLE DES DIFFÉRENTES INFORMATIONS SENSORIELLES DANS L'ATTRAGTION DE LA FEMELLE EN CESTRUS PAR LE MÂLE CHEZ LES PORCINS
}

\author{
J. P. SIGNORET \\ avec la collaboration technique de Colette Lavenet et P. Orgeur
}

Station de Physiologie de la Reproduction. Centre de Recherches de Tours, I. N. R. A., 37380 Nouzilly

RÉSUMÉ

Les mécanismes sensoriels responsables de l'attraction de la Truie en œstrus par le mâle ont été étudiés par des épreuves de choix dans un labyrinthe en $T$. La truie discrimine aussi bien un mâle d'une femelle, qu'un castrat d'un verrat. Cette orientation sélective n'est pas modifiée lorsque les animaux stimulus ne peuvent être vus, non plus lorsque, par une anesthésie, on élimine leurs émissions sonores et leurs réactions motrices et posturales.

On est ainsi conduit à attribuer à une phéromone l'attraction de la truie en cstrus par le verrat. Les résultats expérimentaux ont montré que la sécrétion préputiale n'est pas à l'origine de l'odeur active, qui peut, par contre, être produite par une femelle ovariectomisée recevant des injections journalières de propionate de testostérone $(75 \mathrm{mg} / \mathrm{j}$ par voie intramusculaire). Ceci nous a conduit à l'hypothèse suivante : les produits de métabolisation des androgènes mis en évidence dans les glandes salivaires pourraient être responsables de cette action; ce qui expliquerait également l'importance du contact naso-nasal dans la séquence du comportement sexuel des porcins.

\section{INTRODUCTION}

Dans la recherche mutuelle des partenaires sexuels, l'activité dư mâle est très apparente ; cependant, la femelle joue également un rôle dont l'importance est souvent déterminante comme l'ont montré des études faites aussi bien chez le Rat (LEMAGNEN, I95I ; CARR et al., I965), les ovins (LINDSAY et RoBINSON, I96I) que chez les porcins (Signoret, 1967). Au cours d'une série d'expériences, nous avons tenté de déterminer le rôle des diverses informations sensorielles dans l'attraction de la femelle en œstrus par le mâle dans l'espèce porcine. 


\section{MATÉRIEL ET MÉTHODES}

$$
\text { I. - Dispositif }
$$

Les mécanismes intervenant dans la recherche réciproque du mâle et de la femelle ont été abordés en adaptant à nos animaux les épreuves de choix classiques pour les animaux de laboratoire. Nous avons utilisé un labyrinthe en T, chacun des signaux à comparer présenté à l'extrémité d'une des branches, l'animal expérimental étant introduit par la troisième branche. Pour éviter l'effet de signaux extérieurs, les limites du labyrinthe étaient constituées, soit par un mur, soit par un grillage doublé d'une rangée de ballots de paille s'élevant à $\mathrm{I}, 5 \mathrm{~m}$ de hauteur.

\section{2. - Mode de présentation des stimuli}

A chaque extrémité des deux branches, une case de $2 \times 2 \mathrm{~m}$ a été construite, séparée du labyrinthe par des barreaux de fer verticaux distants de $10 \mathrm{~cm}$ environ. Pour certaines expériences où le stimulus utilisé ne devait pas être vu, cette séparation était doublée d'une cloison de contreplaqué d'une hauteur de $\mathrm{r}, 8 \mathrm{~m}$.

Lorsque nous avons proposé le choix entre verrat, castrat ou femelle, nous n'avons retenu que des individus n'ayant jamais été antérieurement en contact direct avec les sujets expérimentaux. Pour une même série, les deux animaux stimulus sont restés les mêmes.

Dans certains essais, les deux animaux stimulus étaient anesthésiés pour éviter l'effet éventuel de la manière dont l'animal stimulus réagit à l'approche de la femelle en expérience. Pour cela, nous avons utilisé une injection intramusculaire de butyrophénone ("Eucalmyl " laboratoire Sovetal, Paris). Ce produit, injecté à la dose de $20 \mathrm{mg} / \mathrm{ro} \mathrm{kg} \mathrm{de} \mathrm{poids} \mathrm{vif,} \mathrm{immobilise} \mathrm{en} \mathrm{ro-}$ I 5 minutes le sujet qui demeure couché et sans réponse aux stimulations extérieures pendant 2 à 3 heures.

\section{3. - Enregistrement}

La position de l'animal expérimental dans le labyrinthe a été enregistrée en continu par un actographe mis en ceuvre par l'observateur.

$$
\text { 4. - Animaux }
$$

Dix-huit truies adultes de type Large White ovariectomisées ont été utilisées pour ces expériences. La réceptivité sexuelle était induite par injection intramusculaire de I mg de benzoate d'œstradiol. Dans ces conditions, les réactions des femelles sont identiques à celles observées lors de l'œstrus naturel (SIGNORET, I967). Ce traitement était répété tous les 21 jours, délai analogue à la durée moyenne du cycle œstrien de cette espèce. La réceptivité sexuclle était vérifiéc par présentation à un mâle avant les essais. Les animaux ont été divisés en trois groupes pour lesquels les injections étaient décalées d'une semaine, de façon à disposer simultanément d'un groupe de femelles en oestrus et de deux groupes témoins. Les essais ont eu lieu dans ces conditions chaque semaine du lundi au vendredi. Dans l'une des expériences ont été utilisées 6 truies rendues anosmiques, par l'ablation chirurgicale des bulbes olfactifs (SIGNORET et MAUleoN, 1962).

\section{RÉSULTATS}

\section{I. - Infuence de l'état hormonal de l'animal stimulus}

Nous avons cherché à établir dans quelle mesure les signaux émis par le mâle et attirant la femelle en cestrus étaient sous contrôle endocrinien. Nous avons pour cela opposé dans le labyrinthe, soit un mâle à une femelle, soit un mâle intact à un castrat. 
Les résultats présentés dans le tableau I, montrent que la Truie en ostrus discrimine un mâle d'une femelle aussi bien qu'un verrat d'un castrat. Il apparaît donc que les signaux responsables de ce phénomènes sont, en partie tout au moins, sous contrôle des androgènes,

\section{TABLEAU I}

Temps passé par des truies dans un labyrinthe en $T$ à proximité d'un mâle intact opposé à une femelle ou à un castrat

\begin{tabular}{|c|c|c|c|}
\hline Stade physiologique & Nombre & \multicolumn{2}{|c|}{ Temps moyen en secondes pour une épreuve de $5 \mathrm{mn}$} \\
\hline $1_{\text {re }}$ série expérimentale & \multirow{3}{*}{203} & vers le inâle & vers la femelle \\
\hline \multirow[t]{2}{*}{ Hors œstrus } & & $57,76 \pm 3,98$ & $71,13 \pm \quad 5,24$ \\
\hline & & \multicolumn{2}{|c|}{ * } \\
\hline Estrus & 37 & $194,86 \pm 14,52$ & $46,27 \pm 10,78$ \\
\hline 2e série experimentale & & vers le mâle intact & vers le mâle castré \\
\hline \multirow[t]{2}{*}{ Hors cestrus } & \multirow[t]{2}{*}{180} & 79,51 土 6,96 & $102,45 \pm$ \\
\hline & & ** & \\
\hline \multirow[t]{2}{*}{ Estrus } & \multirow[t]{2}{*}{99} & $170,12 \pm 8,76$ & $76,12 \pm$ \\
\hline & & ** & \\
\hline
\end{tabular}

** $\mathrm{P}<0,01 ; \quad * \mathrm{P}<0,05$

L'attraction de la femelle en ostrus, résulte de signaux émis par le mâle, soit passivement (odeur ou aspect général de l'animal), soit activement (émissions sonores, parades ou réponses motrices diverses lors de l'approche de la femelle).

\section{2. - Signaux émis activement par le mâle}

Si l'on veut supprimer l'ensemble de ces signaux, on est contraint d'éliminer non seulement les émissions sonores mais aussi les postures et les réactions motrices diverses. Pour ceci, nous avons réalisé une anesthésie des animaux stimulus.

Les résultats obtenus ont montré que cette intervention ne modifie d'aucune manière la réaction des femelles expérimentales (tabl. 2).

Les seuls signaux intervenant ici sont donc la vue de l'animal et son odeur. Bien que la castrastion à l'âge adulte n'ait pratiquement pas changé 1'aspect extérieur du verrat, nous avons cherché à savoir si l'odeur seule était suffisante lorsque les animaux stimulus ne pouvaient être vus. Nous avons donc fait subir à nos femelles 
expérimentales des épreuves de choix entre I verrat intact et I castrat ne pouvant être vus de l'intérieur du labyrinthe (isolés par une cloison de contreplaqué). Un jour sur deux, les deux animaux stimulus ont été anesthésiés.

\section{TABLEAU 2}

Temps passé par des truies dans un labyrinthe en $T$ à proximité d'un mâle intact ou d'un castrat anesthésiés

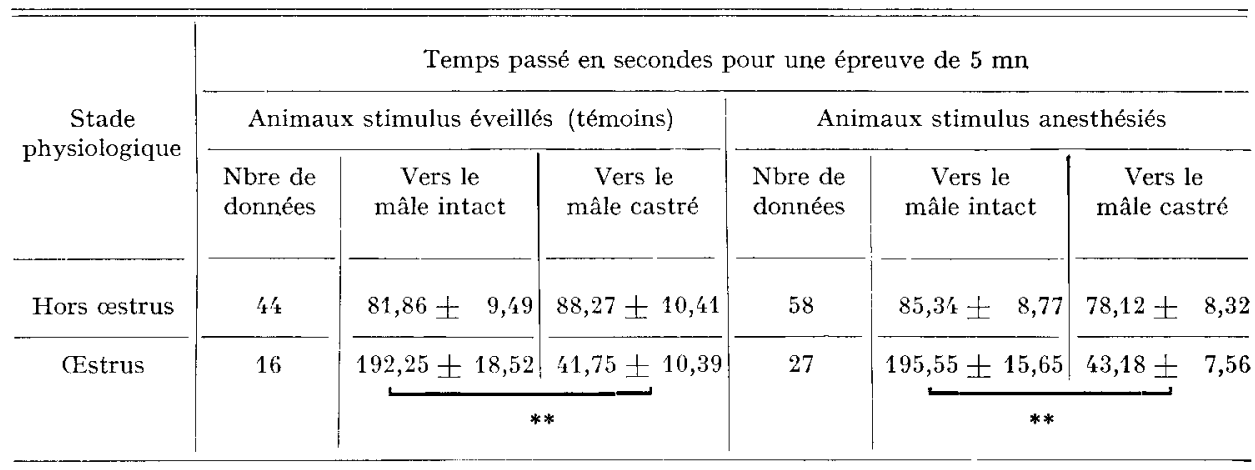

$* * \mathrm{P}<0,01$.

Les résultats obtenus nous ont montré que J'absence de tout signal visuel, ainsi que de 1'activité propre des animaux stimulus, et en particulier des émissions sonores, ne modifie pas la capacité de discrimination de la truie en oestrus (tabl. 3). Les informations olfactives ont donc vraisemblablement un rôle prédominant.

\section{TABL,EAU 3}

Temps passé par des truies dans un labyrinthe en $T$ à proximité d'un male intact ou d'un castrat.

Effet de la suppression des signaux visuels émis par les animaux stimulus et de l'anesthésie de ceux-ci

\begin{tabular}{|c|c|c|c|c|c|c|}
\hline \multirow{3}{*}{$\begin{array}{c}\text { Stade } \\
\text { physiologique }\end{array}$} & \multicolumn{6}{|c|}{$\begin{array}{l}\text { Animaux stimulus non visibles } \\
\text { Temps passé en secondes pour une épreuve de } 5 \mathrm{mn}\end{array}$} \\
\hline & \multicolumn{3}{|c|}{ Témoins éveillés } & \multicolumn{3}{|c|}{ Anesthésiés } \\
\hline & $\begin{array}{l}\text { Nbre de } \\
\text { données }\end{array}$ & $\begin{array}{l}\text { Vers le } \\
\text { mâle intact }\end{array}$ & $\begin{array}{l}\text { Vers le } \\
\text { mâle castré }\end{array}$ & $\begin{array}{l}\text { Nbre de } \\
\text { données }\end{array}$ & $\begin{array}{l}\text { Vers le } \\
\text { mâle intact }\end{array}$ & $\begin{array}{l}\text { Vers le } \\
\text { mâle castré }\end{array}$ \\
\hline Hors cestrus & 29 & $60,07 \pm 9,40$ & $58,69 \pm 10,05$ & 29 & $48,97 \pm 9,74$ & $55,79 \pm 11,83$ \\
\hline Estrus & 13 & $\underbrace{159,54 \pm 25,82}_{*}$ & $38,62 \pm 10,37$ & 14 & $\underbrace{148,14 \pm 24,99}_{*}$ & $64,14 \pm 19,52$ \\
\hline
\end{tabular}

** $\mathrm{P}<0,01$. 


\section{3. - Action des informations olfactives}

Nous avons observé l'effet de la suppression des informations olfactives en nous plaçant dans les conditions où la discrimination est la plus nette, c'est-à-dire en opposant un mâle et une femelle dans une épreuve de choix. Les six truies privées de l'odorat ont présenté lors de l'œstrus une légère préférence pour le mâle mais la différence est restée faible et n'a pas atteint le seuil de la signification statistique (tabl. 4).

\section{TABLEAU 4}

Effet de l'ablation des bulbes olfactifs sur l'orientation des truies dans un labyrinthe en $T$, vers un mâle ou une femelle

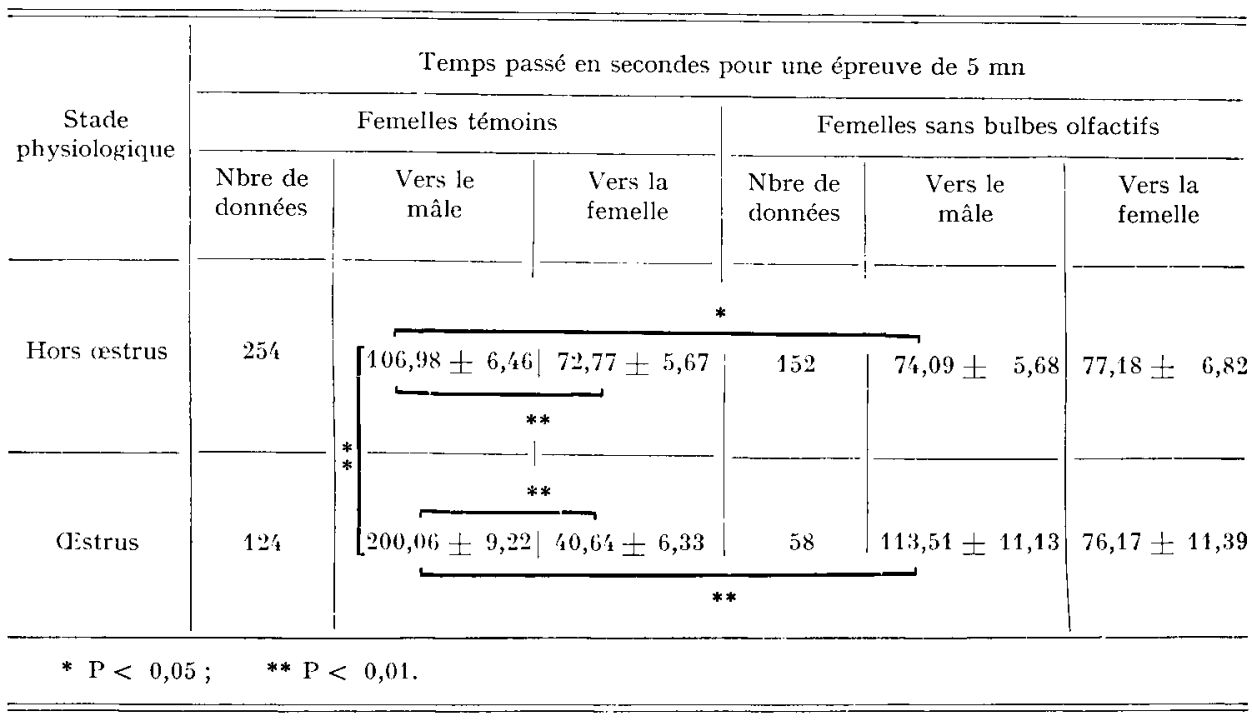

Ainsi, donc, même dans les conditions les plus favorables à une discrimination, la suppression de l'odorat par ablation des bulbes olfactifs réduit considérablement la capacité de recherche du mâle par la femelle.

Nous avons cherché à identifier l'origine de l'odeur impliquée dans l'attraction de la femelle en œstrus. Pour cela nous avons utilisé l'odeur de la sécrétion préputiale qui s'était révélée active pour faciliter la réponse posturale de la femelle en œstrus (Signoret et du MEsnil du Buisson, ig6r).

Nous avons opposé un verrat intact soit à un verrat castré arrosé de sécrétion préputiale d'un autre mâle intact, soit à un verrat castré. Pour éviter l'effet de signaux visuels, les deux animaux stimulus étaient placés derrière une cloison de contreplaqué. Enfin, pour éviter l'influence des émissions sonores, un essai sur deux a eu lieu avec les deux verrats anesthésiés.

L'utilisation d'odeur sur le verrat castré n'a en aucun cas augmenté le temps passé à proximité de lui par la femelle en œstrus. L'existence d'une attraction significative mais hors de l'œstrus pourrait être le reflet d'une recherche de contact avec un animal connu, portant une odeur inhabituelle. Cependant, ceci n'a été observé que sans anesthésie et disparaît lorsque les femelles sont en cestrus (tab1. 5). 


\section{TABLEAU 5}

Effet de l'odeur de sécrétion préputiale sur l'orientation d'une truie dans un labyrinthe en $T$ où lui est proposé le choix entre un male intact et un castrat

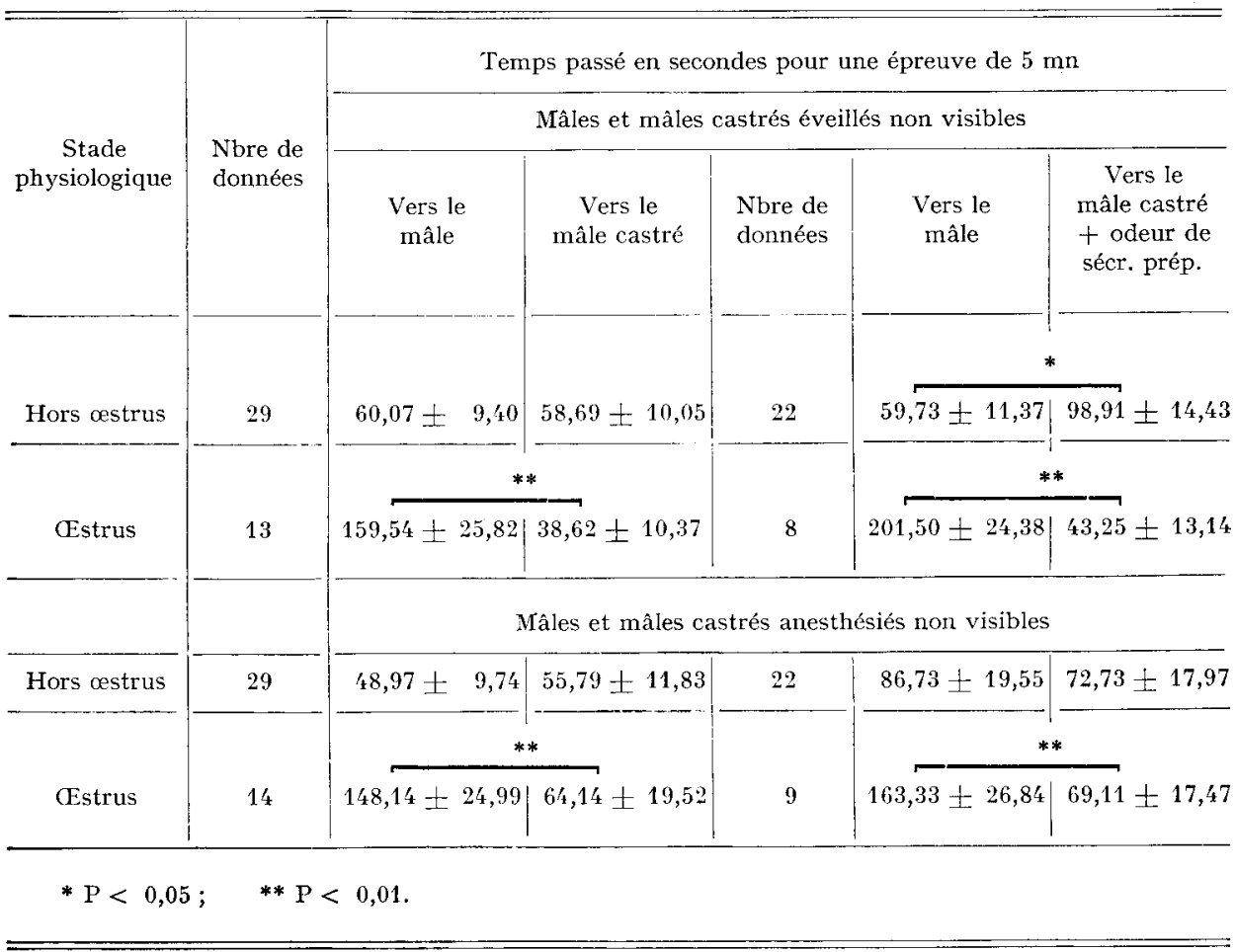

\section{TABLEAU 6}

Temps passé par des truies dans un labyrinthe en $T$ à proximité d'une femelle "androgénisée "opposée à une femelle ovariectomisée témoin

\begin{tabular}{|c|c|c|c|}
\hline \multirow{2}{*}{$\begin{array}{c}\text { Stade } \\
\text { physiologique }\end{array}$} & \multicolumn{3}{|c|}{ Temps passé en secondes pour une épreuve de $5 \mathrm{mn}$} \\
\hline & $\begin{array}{c}\text { Nombre } \\
\text { de données }\end{array}$ & $\begin{array}{l}\text { Vers la femelle } \\
\text { androgénisée }\end{array}$ & $\begin{array}{l}\text { Vers la femelle } \\
\text { témoin }\end{array}$ \\
\hline & & & \\
\hline \multirow[t]{2}{*}{ Hors cestrus } & 270 & 43,82 & 81,22 \\
\hline & & \multicolumn{2}{|c|}{ ** } \\
\hline Estrus & 109 & $173,1_{1}^{\prime} t_{1}$ & 38,01 \\
\hline$* * \mathrm{P}<0,0$ & & & \\
\hline
\end{tabular}


L'origine de l'odeur qui attire la truie en œstrus pouvait donc être un autre produit résultant de l'action des hormones stéroïdes sexuelles. S'il en était ainsi, une femelle ovariectomisée traitée aux androgènes pourrait provoquer, comme un mâle, l'attraction des truies en œstrus dans la mesure où elle possèderait les mêmes enzymes de dégradation de ces stéroïdes et les mêmes organes de sécrétion. Pour mettre à l'épreuve cette hypothèse, nous avons préparé une truie ovariectomisée en lui faisant subir des injections journalières de $75 \mathrm{mg}$ de propionate de testostérone. Les résultats (tabl. 6) montrent que la valeur d'attraction d'une truie traitée aux androgènes est comparable à celle du mâle. Il existe donc une substance phéromone responsable de ce mécanisme et qui est produite sous l'action des androgènes par d'autres organes que ceux spécifiques du mâle.

\section{DISCUSSION ET CONCLUSION}

Les phéromones jouent un rôle déterminant dans l'interattraction des partenaires sexuels puisque les informations fournies par l'odorat sont nécessaires et suffisantes pour l'organisation de cette phase du comportement sexuel. L'odeur spécifique des mâles peut avoir des origines diverses. A côté de glandes spécialisées, il peut y avoir une émission de produits odorants spécifiques avec les déjections; c'est le cas par exemple des crottes de lapins, où le produit des glandes anales s'ajoute aux excréments et joue un rôle important dans le marquage territorial (MykyTowIcz, I966). L'urine contient également des produits odorants susceptibles d'avoir un rôle dans les relations inter-individuelles. Des phéromones agissant aussi bien dans le domaine de la reproduction que dans celui des relations sociales ont été mises en évidence dans l'urine chez le mâle des souris.

Chez le mâle des suidés, deux sources d'odeur caractéristique ont été mises en évidence. Le verrat possède une sécrétion préputiale abondante, d'une odeur très forte et caractéristique. La substance chimique responsable de cette odeur a été isolée pat Patterson (I968 a) : il s'agit du $5 x$-androst-I6-ène-3-OL. Cependant, nous avons vu que cette phéromone ne peut rendre compte de l'interattraction que nous avons observée. PatTERSON ( $\mathrm{g} 968 \mathrm{~b}$ ) a isolé également un dérivé du métabolisme des androgènes dans les glandes salivaires sous-maxillaires du verrat. Ce composé d'odeur musquée, se retrouve dans la salive et semble responsable de l'odeur caractéristique de l'haleine du verrat. L'existence de ces deux produits et leur différente localisation chez l'animal ont été confirmées par le travail de CLAUS (I970).

L'existence de ces deux odeurs sexuelles pouvant être émises au niveau de l'orifice préputial d'une part, et dans l'haleine d'autre part, pourrait expliquer l'importance comparable des contacts naso-nasal et naso-génital dans la séquence comportementale (SIGNORET, I970).

Il est possible que la phéromone émise dans la salive, et diffusée par l'haleine du mâle, puisse participer à l'attraction de la femelle réceptive. Cette hypothèse est étayée par les résultats de PERRY et al. (I972) qui montrent que l'ablation des glandes salivaires sous-maxillaires d'un verrat perturbe les réactions des femelles à son égard. 
Il apparait donc que deux phéromones interviennent dans l'organisation du comportement sexuel des porcins : à côté de celles qui rendent compte de l'attraction de la truie en cestrus par le verrat, nous avons antérieurement mis en évidence le rôle de l'odeur de la sécrétion préputiale pour faciliter l'apparition de la réaction posturale d'immobilisation de la femelle (Signoret, du Mesnil du Buisson, I96I ; MELrose et al., I97I). Dans ce cas, cependant, d'autres informations sensorielles, acoustiques ou visuelles, ont avec elles une action cumulative : en l'absence d'informations olfactives, la vue et surtout l'ouie peuvent exercer une suppléance permettant l'organisation des réponses comportementales.

Il est à remarquer que notre étude de l'ensemble du comportement sexuel des porcins ne nous a pas permis de mettre en évidence la production de phéromones par la femelle. Si l'on ne peut exclure l'éventualité de leur existence, il est possible d'affirmer qu'elles ne joueraient en tout cas qu'un rôle accessoire, contrairement à ce qui se passe dans d'autres espèces comme les rongeurs (LEMAGNEN, I95I ; CARR et al., I965) ou les primates (Michael et KeVERnE, I968).

Reçu pour publication en juin 1974.

\author{
SUMMARY \\ ROI,E OF SOME SENSORY STIMUL, \\ IN THE ATTRACTION OF THE GSTROUS SOW BY THE BOAR
}

The sensory stimuli involved in the attraction of the ostrous sow by the boar were studied in T-maze experiments. The orientation of the female to the male is similar when, she is opposed in the T-maze to a female or a castrate ( 194 vs 46 sec and 192 vs 4 I sec respectively in a 300 sec test). Such a discrimination is unmodified when both stimulus animals cannot be seen (I59 vs $38 \mathrm{sec}$ ) and/or were anesthetized to eliminate the emission of acoustic signal and the postural and motor reactions ( $195 \mathrm{vs} 43 \mathrm{sec}$ ). These results lead to the conclusion that a pheromone probably accounts for the attraction of the œstrous sow by the boar. The preputial secretion, sprayed on a castrated male does not improve his attractive value when opposed to an intact male in the T-maze (2OI vs $43 \mathrm{sec}$ ). However, the I/M injection of a daily dose of $75 \mathrm{mg}$ of testosterone propionate to an ovariectomized sow increases her attractive value, when opposed to a control female in the T-maze, to a level similar to that of the male ( $173 \mathrm{vs} 38 \mathrm{sec}$ ).

Among the other possible sources of pheromone, the hypothesis was emitted of a role of the androgen metabolites in the submaxillary salivary gland which can account for the frequency of naso-nasal interaction in the precopulatory sequence in the pig.

\title{
RÉFÉRENCES BIBLIOGRAPHIQUES
}

Carr W. J., Loeb L. S., Dissinger M. L., 1965. Responses of rats to sex odors. J. Comp. Physiol. Psychol., 59, 270-377.

Claus R., r97o. Bestimmung von Testosteron und 5- $\alpha$-androst-I6-en-3-on, einem Ebergeruchsstoff, bein Schweinen. Diss. Fas. Landtirts, München.

Lemagnen J., I95I. Étude des phénomènes olfacto-sexuels chez le Rat blanc. Variations selon leur état sexuel de la réponse des mâles à l'odeur de la femelle et réponse des femelles à l'odeur du mâle. $C . R$. Soc. Biol., 145, 857-86r.

Lindsay D. R., Robinson T. J., I96r. Studies on the efficiency of mating in sheep. J. Agric. Sci. (Camb), 57, I37-145. 
Melrose D. R., Reed H. C. B., Patterson R. L. S., I97I. Androgen steroids associated with boar odour as an aid to the detection of cstrus in pig artificial insemination. B. Vet. J., $127,497$.

Michael R. P., Keverne E. B., I968. Pheromones in the communication of sexual status in primates. Nature, 218, 746-749.

MYкүтоWYсz R., I966. Observations on odoriferous and other glands in the Australian wild rabbit Oryc. Cun., and the hare, Lep. Europ. I. The anal gland. C. S. I. R. O. Wildl. Res., 11, II-29.

Patterson R. L. S., Ig68 a. A $5 \alpha$-Androst-I6-en-3-one : compound responsible for taint in boar fat. J. Sci. Fd. Agric., 19, 3I-38.

Patterson R. L. S., I968 $b$. Identification of $3 \alpha$-hydroxy-5 $\alpha$-androst-I 6 -ene as the musk odour component of boar submaxillary salivary gland and its relationship to the sex odour taint in pork meat. J. Sci. Fd. Agric., 19, 434-438.

Perry G. C., Patterson R. L. S., Stinson G. C., I972. Submaxillary salivary gland involvement in porcine mating behaviour. VII' Cong. Int. Reprod. anim. Insem. artif., München.

Signoret J. P., du Mesnil du Buisson F., ig6r. Étude du comportement de la Truie en œstrus. IV Cong. int. Reprod, anim., La Haye, I7I-I 75.

Signoret J. P., Mauleon P., I962. Action de l'ablation des bulbes olfactifs sur les mécanismes de la reproduction chez la Truie. Ann. Biol. anim. Bioch. Biophys., 2, 167-174.

Signoret J. P., ig67. Attraction de la femelle en œestrus par le mâle chez les porcins. Rev. Comp anim., 4, 10-22.

Signoret J. P., 1970. Reproductive behaviour of pigs. J. Reprod. Fert., sup. 11, I05-II7. 